\title{
VIABILIDAD DEL HÁBITAT Y DEL REFORZAMIENTO POBLACIONAL DE CAPRA PYRENAICA EN LA RESERVA NACIONAL DE CAZA DE OS ANCARES
}

\section{Habitat and population reinforcement of Capra pirenaica in the National Game Reserve of Os Ancares}

\author{
C. Prada ${ }^{1} \&$ J. Herrero ${ }^{2}$ \\ 1 Ega Consultores en Vida Silvestre SLPU. Sierra de Vicort, 31, 1. ${ }^{\circ}$ A - E-50003 Zaragoza \\ egasl@arraki.es \\ 2 Área de Ecología. Departamento de Ciencias Agrarias y del Medio Natural. Escuela Politécnica \\ Superior. Universidad de Zaragoza. E-22718 Huesca. herreroj@unizar.es
}

\begin{abstract}
RESUMEN.- La cabra montés, Capra pirenaica, ha sido reintroducida en la Cordillera Cantábrica en León (Riaño y Ancares). Con el fin de evaluar la viabilidad del hábitat y del reforzamiento poblacional en la Reserva Nacional de Caza de Os Ancares (RNCA, Lugo) llevamos a cabo: (i) una reconstrucción de su historia natural en el NO de la Península Ibérica; (ii) una recolección de citas y determinación de la distribución actual en Ancares; (iii) el análisis del uso del espacio a partir de revisión de experiencias similares; (iv) un desarrollo de cartografía para definir su hábitat potencial y v) una zonificación de la RNCA en función de la calidad del hábitat. Los resultados indican la existencia de un área adecuada de 1.250 ha que podría albergar una población de 40 ejemplares considerando una densidad de 3 cabras km-2. La gestión de esta población debería estar coordinada con la de la parte leonesa del macizo, de mayor calidad y superficie que la parte lucense. El crecimiento actual de la población existente permitirá a la especie colonizar todo el macizo de forma natural.
\end{abstract}

Palabras clave: Matorralización, epizootias, ungulados, Canis lupus, abandono rural, Lugo.

ABSTRACT.- Iberian wild goat Capra pyrenaica has been re-introduced in the Cantabrian Mountains in Leon province (Riaño and Ancares). To evaluate habitat and restocking suitability in the National Game Reserve of Os Ancares (NGRA, 


\begin{abstract}
Lugo province) we: (i) undertook a reconstruction of its natural history in the NW of the Iberian Peninsula; (ii) gathered data on its current distribution in Ancares; (iii) analyzed its habitat use though a review of similar experiences; (iv) developed a cartography to define its potential habitat, and $y v$ ) drew a zoning of the NGRA following habitat quality. Results indicate the existence of suitable habitat in 1,250, which could host 40 animals considering a density of 3 goat $\mathrm{km}^{-2}$. The management of this population should we coordinated with the leonese side of the massif, of higher quality and surface rather than the Lugo's slope. The increase of the current population will allow the species to colonize naturally the whole massif.
\end{abstract}

Keywords: Scrub encroachment, epizootics, ungulates, Canis lupus, rural abandonment, Lugo.

\title{
1. Introducción
}

La cabra montés Capra pyrenaica (ver García-González, 2011, para una reciente revisión) es un ungulado de montaña endémico de la Península Ibérica, a la que llegó presumiblemente atravesando los Pirineos durante la glaciación de Würm. Hasta el siglo XIX se distribuía prácticamente por todas las áreas de hábitat favorable de la Península Ibérica (Cabrera, 1911), pero desde entonces ha sufrido una profunda regresión de sus poblaciones, con varios cuellos de botella demográficos que han provocado una elevada consanguinidad y una consecuente pérdida de variabilidad genética de sus poblaciones (Amills et al., 2004). En la actualidad se encuentra en pleno proceso expansivo, debido a su recuperación natural y a los traslados (Pérez et al., 2002). Está clasificada como de Preocupación Menor (Least Concern, LC) por la Unión Mundial para la Conservación de la Naturaleza (UICN) (Herrero y Pérez, 2008).

De las cuatro subespecies descritas por Cabrera en 1911 sobreviven dos, C.p.hispanica, que ocupa el E de la Península y C. p. victoriae, el W (Pérez et al. 2002). C. p. lusitanica (Cabrera, 1911), que ocupaba la Sierra de Gerês en Portugal (Almança 1992) y algunas montañas gallegas (Seoane, 1866), se extinguió a finales del siglo XIX; y C. p. pyrenaica, que vivía en el Pirineo, lo hizo a principios del XXI (García-González y Herrero, 1999). La sistemática propuesta por Cabrera (1911), basada en características morfológicas externas como el diseño del pelaje y la forma de los cuernos, ha sido cuestionada (Couturier, 1962; Clouet, 1979; Manceau et al., 1999). Hay que subrayar que hoy en día el análisis filogeográfico, además del genético y biométrico, aporta nuevas herramientas de análisis sobre las poblaciones presentes y pasadas de la especie (García-González, 2011).

Diversas sueltas de cabra montés realizadas en los últimos años del siglo XX y primeros del XXI en la Sierra de Ancares han desembocado en que 
comienzan a verse algunos ejemplares en la Reserva Nacional de Caza de Os Ancares (RNCA), en Lugo. Esta nueva situación, unida a su catalogación como especie cinegética, impulsa la necesidad de reflexionar sobre la posibilidad de mantener una población de cabra montés en la RNCA con vistas a su posible aprovechamiento cinegético.

La finalidad de este artículo es por tanto valorar la idoneidad de la RNCA como hábitat para la cabra montés en el marco de la Sierra de Ancares en su conjunto y de la Cordillera Cantábrica en general. Para ello se han planteado los siguientes objetivos: (i) descripción de la situación de la cabra montés en el NO de la Península Ibérica; (ii) recolección de citas y determinación de la distribución actual de la especie en el macizo; (iii) análisis del uso del espacio de la cabra montés y revisión de experiencias similares; (iv) desarrollo de cartografía temática para definir las principales características ambientales que condicionan el hábitat de la cabra montés y (v) zonificación de la RNCA en función de la calidad del hábitat para la especie.

\section{Material y métodos}

Se llevó a cabo una revisión bibliográfica sobre la cabra montés en los siguientes aspectos: distribución histórica en Galicia y la Cordillera Cantábrica; uso y selección de hábitat de la especie y de otros ungulados silvestres y conocimiento científico y de gestión. En la revisión se han tenido en cuenta artículos científicos, divulgativos, informes técnicos, libros históricos y artículos de prensa.

Se prospectaron las zonas más interesantes para la cabra de la RNCA y, en general, de la Sierra de Ancares, tanto la parte leonesa como la gallega y se realizaron entrevistas a guardas y técnicos de la RNCA y de la RRCA.

Para evaluar la aptitud geográfica de la RNCA en función de los requerimientos ecológicos de la cabra montés se realizó un análisis cartográfico con la ayuda de un Sistema de Información Geográfica (SIG). Se usaron las variables espaciales y umbrales de referencia descritos en los apartados 4.3 y 4.4. Se ha trabajado con diversa información geográfica digital procedente del Centro Nacional de Información Geográfica (CNIG): Modelo Digital de Elevaciones (MDE), ortofotografías aéreas pertenecientes al Plan Nacional de Ortofotografía Aérea (PNOA), Corine Land Cover 2006 e información cartográfica y topográfica en formato vectorial perteneciente a la serie MTN25. También se han usado otras capas de información y bases de datos espaciales públicas como los límites municipales (IGN) o la Red Natura 2000. Los datos de localizaciones de individuos o lugares de suelta se han digitalizado directamente en pantalla a partir de las comunicaciones 
personales recogidas. Se ha trabajado con proyección UTM, huso $29 \mathrm{~T}$ y datum ETRS89.

El análisis de la información geográfica consistió en la superposición y suma de los mapas temáticos creados con las variables seleccionadas reclasificadas según los criterios que se describen en el apartado 4.4. La zonificación final se realizó de forma manual agrupando las zonas similares resultantes.

\section{3. Área de estudio}

La RNCA se sitúa en el límite oriental de la provincia de Lugo, en las estribaciones occidentales de la Cordillera Cantábrica, ocupando una superficie de 8.826 ha. Es Zona de Protección del Oso Pardo, Lugar de Importancia Comunitaria (LIC), Zona de Especial Protección para las Aves (ZEPA) y Reserva de la Biosfera.

Su relieve está constituido por un eje montañoso principal, que concentra las mayores alturas de la Reserva, y profundos valles transversales divididos por sierras. Las pendientes son muy elevadas originando grandes desniveles en muy poco espacio, aunque no es habitual la aparición de grandes aforamientos rocosos. La zona está sometida a un clima caracterizado por las elevadas precipitaciones e inviernos rigurosos. Presenta un paisaje históricamente modelado por la acción de hombre formando un mosaico de cultivos y bosques que en los últimos años, a partir del éxodo rural, ha evolucionado hacia un mosaico de bosques (robledales Quercus robur, abedulares Betula celtiberica, acebedas Ilex aquifolium, castañares Castanea sativa), amplias zonas de matorral (brezales Erica sp., piornales Cytisus purgans, enebrales Juniperus communis) y pastizales en las zonas más altas.

En la fauna destaca el oso pardo Ursus arctos, liebre de piornal Lepus castroviejoi y perdiz pardilla Perdix perdix. Los ungulados están representados por el corzo Capreolus capreolus, cuya población motivó la creación de la RNCA, el jabalí Sus scrofa, el rebeco cantábrico Rupicapra pyrenaica parva, el ciervo Cervus elaphus y la cabra montés Capra pyrenaica, los tres últimos procedentes de sueltas. La Reserva cuenta también con una importante población de lobo Canis lupus (Llaneza et al., 2011).

La RNCA se localiza íntegramente en la zona oriental del municipio de Cervantes, con una densidad humana de 6,1 habitantes $\mathrm{km}^{-2}$, de las más bajas de Galicia (IGE 2010). La ganadería, agricultura y aprovechamiento forestal constituyen, junto con los servicios en los núcleos más turísticos, los ejes de la economía de la Reserva. 


\section{Resultados}

\subsection{Paleontología extinción y cronología de los traslados en la Cordillera Cantábrica y Galicia}

Existen evidencias de presencia de cabra montés en la Cordillera Cantábrica por un periodo prehistórico dilatado, comprendido entre el Pleistoceno y el Holoceno. En Asturias, en la Caverna de la Peña de Candamo, se encontraron a principios del siglo XX grabados esquemáticos, uno de ellos de una cabeza de cabra montés perteneciente al Paleolítico Superior (Hernández-Pacheco, 1919).

Ángel Cabrera (1911) recoge en un mapa la distribución de la cabra montés en la Península Ibérica a principios del s.XX y en el pasado. La especie presentaba una distribución continua por toda la Cordillera Cantábrica y macizos Galaico-leoneses a principios del siglo XIX, aunque esta distribución no se corresponde con las escasas citas históricas de cabra montés en la Cordillera Cantábrica. La primera cita registrada en la Cordillera Cantábrica es de 1801 y la última de 1858, ambas en el concejo de Cabrales (Uría, 1976). Entre ellas, Arribas (2004) aporta tres citas en Asturias (1812 Lena, 1827 Amieva, 1852-4 Cabrales). Comunicaciones personales entre HernándezPacheco y Uría, recogidas por este último (1976) datan la presencia de cabras monteses en algunas montañas de León hasta mediados del siglo XIX.

La otra población conocida cercana a Ancares es la que habitaba en la frontera luso-orensana. Almança (1992) describe la evolución de los últimos ejemplares de esta población de C.p. Iusitánica que habitaba en la Serra do Gêres. En 1870 se cifra la población en 7 ejemplares, que aumenta hasta los 12 vistos en 1886 en la cuenca del río Caldo, en la frontera española. En 1890 se captura el último ejemplar vivo, durante 1891 se encontraron dos individuos muertos y en 1892 se produce el último avistamiento conocido.

Desde su extinción en la Cordillera Cantábrica ha habido varios intentos de reintroducción fallidos con ejemplares procedentes de Gredos: en 1928 a partir de una petición expresa del Rey Alfonso XIII (Conde de Yebes, 1943); en 1959 con ejemplares criados en un cercado (Marqués de Valdueza, 1988) y entre 1957 y 1962 a partir de otra suelta (Ortuño y de la Peña, 1977). En los años 80 del siglo XX se planteó la reintroducción de la cabra montés en la actual Reserva Regional de Caza de Riaño (RRC Riaño), en el norte de León. Se seleccionó una zona con un hábitat favorable, con facilidades para su cercado, y se liberaron 30 individuos de C. p. victoriae procedentes de la actual RRC de las Batuecas (Salamanca), 15 hembras y 15 machos (Peral, 1993). Posteriormente este núcleo fue reforzado con ejemplares procedentes de la 
Reserva Regional de Caza de Gredos y pertenecientes a la misma subespecie (Pérez et al., 2002). Esta vez la reintroducción prosperó y actualmente hay un población de varios cientos de cabras que comienzan incluso a dejarse ver por la vertiente asturiana (J.C. Peral com. pers.).

En Ourense se han llevado a cabo dos traslados. El primero en 1992 a un cercado del Parque Natural do Invernadeiro con ejemplares de Las Batuecas (Pérez et al., 2002). El segundo, a otro cercado del Parque Natural Baixa Limia e Serra do Xurés (Moço et al., 2006). Estos ejemplares escaparon y ocuparon a la vez este parque y el colindante Parque Nacional de Peneda Gerês en Portugal, representando la primera presencia de cabra montés para el país desde el siglo XIX tras la extinción de la subespecie lusitanica (Moço et al., 2006).

En la RRC de Ancares, en León, entre los años 1999 y 2001 se han soltado un total de 39 ejemplares procedentes de la RRC de Riaño (A. Martino com. pers.). La primera de ellas de 9 ejemplares durante la primavera de 1999 (J.C. Peral com. pers. a Pérez et al. 2002). Actualmente la población de cabra montés en esta RRC cuenta con un número cercano a 300 individuos con una tasa de incremento anual de la población en torno al 20\% anual (A. Martino com. pers.).

En la contigua RNCA se han realizado entre los años 2002 y 2004 al menos 3 sueltas con ejemplares procedentes de Invernadeiro y el Parque Natural de Baixa Limia e Serra do Xurés con escaso éxito (J. Amigo, J.E. Díaz, J.F. Layna com. pers.)

Existen por tanto en la actualidad cuatro núcleos de cabra montés en el noreste peninsular ibérico: Ancares y Riaño, en la Cordillera Cantábrica; el núcleo transfronterizo de Xurés - Geres y el cercado de Invernadeiro. Todos ellos con un origen último en la Sierra de Gredos (Figura 1).

\subsection{Presencia de cabra montés en la RNCA}

Los primeros ejemplares de cabra montés avistados en la parte lucense de Ancares corresponden a dos machos marcados con radioemisores avistados en Pena Rubia en mayo de 1999 procedentes de la primera suelta que se acababa de realizar en León (J.F. Layna com. pers.). Durante los primeros años del siglo XXI, coincidiendo con las sueltas realizadas en León, se han observado esporádicamente más ejemplares entre la zona de Pena Rubia y la de Tres Bispos, en la línea de crestas que marca el límite provincial (A. Martino com. pers.). Poco después de las sueltas en la RNCA, se vio durante algún tiempo un grupo de hembras en una zona de bosque cercana (M. Rodríguez com. pers.). También se han encontrado varios machos muertos en 


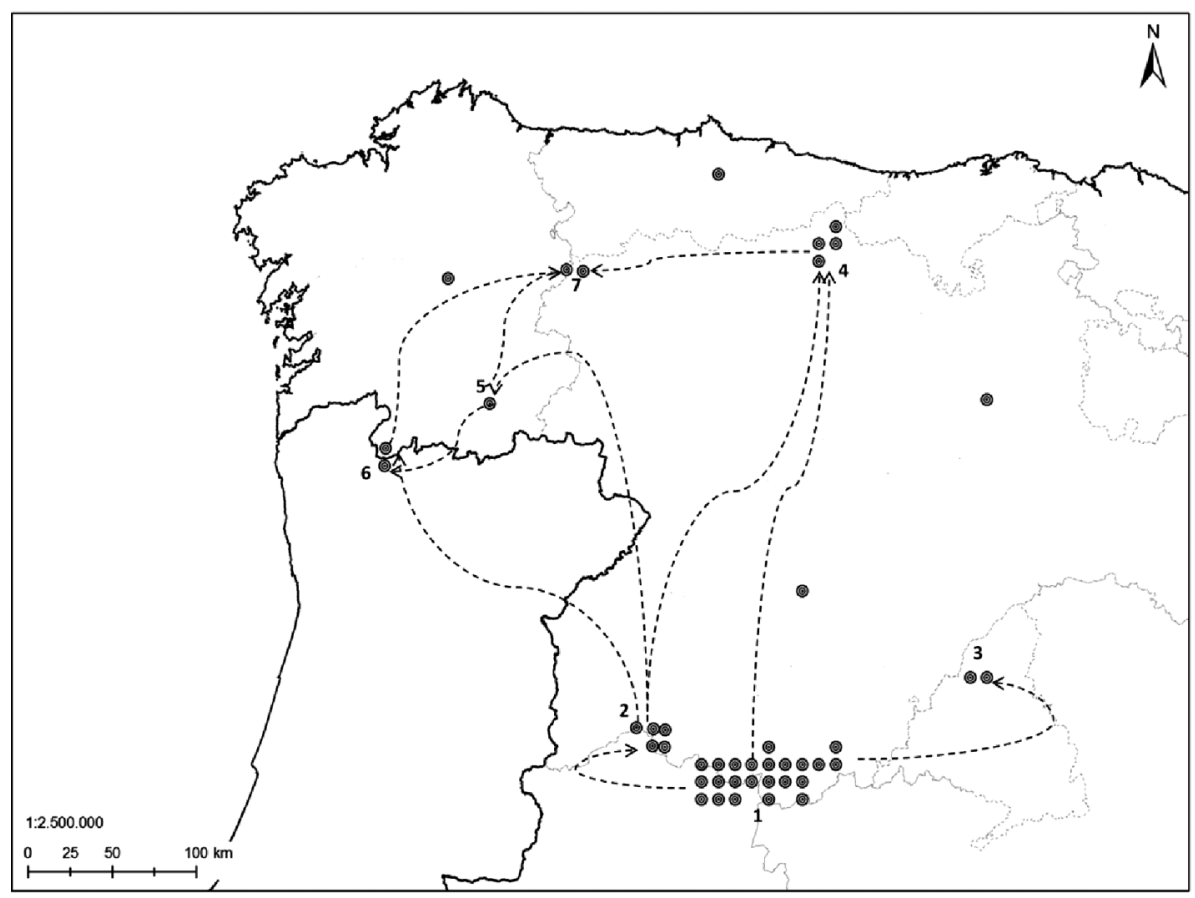

Figura 1. Traslados de cabra montés en el NW ibérico. 1: Gredos; 2: Batuecas; 3: La Pedriza; 4: Riaño; 5: Invernadeiro; 6: Gerês - Xurés; 7: Ancares. Fuentes: Acevedo y Casinello (2009) y este trabajo.

Figure 1. Iberian wild goat translocations in NW Iberia. 1: Gredos; 2: Batuecas; 3: La Pedriza; 4: Riaño; 5: Invernadeiro; 6: Gerês - Xurés; 7: Ancares. Sources: Acevedo and Casinello (2009) and this article.

los últimos años (M. Rodríguez com. pers; J. Amigo y J. E. Díaz com. pers.). Actualmente se producen avistamientos frecuentes en la vertiente sur de la cuerda de Pena Longa, en una zona limítrofe con la RNCA, y más esporádicos en la vertiente oeste del pico Mustallar, dentro de la RNCA. Por último, en 2008 se localizó un grupo de seis hembras sin crías en la zona central de la RNCA, que son avistadas de forma regular desde entonces (J. E. Díaz com. pers.).

\subsection{Revisión de variables que determinan el hábitat de la cabra montés}

Tradicionalmente se ha considerado la altitud como una de las variables que mejor define la adaptación espacial de la cabra montés. Diversos autores 
describen franjas de distribución o cotas mínimas de observación de la especie en distintas áreas de la Península como Cazorla (Fandos, 1991; Alados y Escós, 1996), Sierra Nevada (Escós y Alados 1992; Pérez et. al., 1994); o Sierra Mágina (Palomares y Ruíz Martínez, 1993). En otras especies de caprinos ocurre lo mismo. Fox et al. (1992) localizan los íbices asiáticos Capra ibex sibirica en el Himalaya, en alturas superiores a los $4000 \mathrm{~m}$ y en los Alpes numerosos trabajos sobre íbice alpino Capra ibex ibex determinan altitudes de distribución de la especie que oscilan entre los 1800 y 3000 m (Couturier, 1962; Nievergelt, 1966; Pfeffer y Settimo, 1973; Wiersma, 1983; Francisci et al., 1985; Peracino y Bassano, 1994).

Otros autores como Gauthier et al., (1994) no consideran que la altura determine el hábitat de los caprinos. En esta línea, resultados de varios seguimientos en poblaciones del arco mediterráneo (Prada et al., 2010; Prada et al., 2011) y de la provincia de Zaragoza (García-Serrano et al., 2010) recogen abundantes localizaciones en cotas muy bajas (200-600 m); y en los acantilados de Cerro Gordo entre las provincias de Granada y Málaga estas localizaciones llegan hasta el nivel del mar (Granados et al., 2003).

La única constante ambiental que aparece en los hábitats ocupados en su área de distribución es un relieve con presencia de roca (Fandos, 1991; Alados y Escós, 1996). Las cabras monteses presentan varios caracteres que manifiestan su adaptación a la roca, como el equilibrio o la estructura de la pezuña. Están adaptadas tanto etológica como ecológicamente a todo tipo de roca, independientemente de su naturaleza (Crampe, 1991), resultando un elemento especialmente importante para las hembras y las crías en zonas donde la especie está sujeta a la presencia de depredadores o del hombre (San Segundo y Díez, 1997).

En este sentido la distribución de la cabra en Andalucía se produce mayoritariamente por zonas escarpadas (Granados et al., 2003). Similares conclusiones fueron puestas de manifiesto por Byers (1977) en Capra ibex sibirica o Gauthier et al., (1994) al plantear la reintroducción del íbice alpino en Francia. Peral (1993) relata cómo las primeras observaciones realizadas tras la suelta de cabras en la RRC de Riaño se produjeron en la zona más abrupta e inaccesible del cercado de 2.500 ha donde se soltaron, sobre una superficie de apenas 400 ha.

Para Wiersma (1984) o Gauthier et al., (1994) uno de los factores determinantes para la ocupación del hábitat por el íbice alpino es la orientación, con presencia de laderas de exposición soleada. Choisy (1994) pone de manifiesto que los fracasos en las reintroducciones de íbice alpino en Francia están correlacionados sobre todo con la existencia de pendientes y áreas de invernada. En la Península Ibérica Granados et al. (2003) observan que las exposiciones predominantes en los núcleos montañosos ocupados por 
la cabra montés son las de orientación este y sur, al igual que Alados y Escós (1996) en Sierra Nevada.

Una de las características más importantes de la especie es su gregarismo, generando rebaños de diferente tamaño y composición a lo largo del año (Alados y Escós, 1985) que realizan movimientos trasterminantes altitudinales (Cabrera, 1914; Gonzalez, 1982; Alados y Escós, 1996). Esto requiere una superficie de hábitat adecuado suficiente $\mathrm{o}$ varios interconectados por corredores.

La sensibilidad a las molestias humanas por parte de las cabras monteses es muy variable entre poblaciones. En Sierra Nevada, tanto Alados y Escós (1996) como Cano-Manuel et al., (2008) observaron que se adaptan con facilidad a una elevada presión turística.

La alimentación de la cabra montés ha sido estudiada en diversos macizos montañosos ibéricos: Sierra Nevada (Martínez, 1988a); Cazorla (GarcíaGonzález y Cuartas, 1992; Alados y Escós, 1996); Gredos (Martínez y Martínez, 1987; Martínez 1989); Sierra de Tejeda-Almijara (Martínez, 1988b); Tortosa y Beceite (Martínez, 1994) y Sierra de Montenegro (Palacios et al., Ibañez y Escudero, 1978). Martínez (1992), la clasifica como una especie muy generalista, pastadora o ramoneadora según la oferta alimentaria. Existe un cierto solapamiento de la dieta entre la cabra montés y otros ungulados simpátricos en ambientes mediterráneos (García-González y Cuartas, 1989; Martínez, 1992) y atlánticos (Martínez, 1992).

La competencia no es exclusivamente por el alimento, sino por otros recursos como el espacio, la tranquilidad o el refugio (Fandos et al., 2007). Un efecto derivado del contacto entre ungulados es la transmisión de enfermedades. En este sentido, la que ha causado una mayor mortalidad ha sido la sarna sarcóptica Sarcoptes scabiei, que constituye actualmente una de las principales preocupaciones sanitarias en ungulados silvestres de la Cordillera Cantábrica con poblaciones de rebeco y cabra montés afectadas.

\subsection{Zonificación}

A partir de los resultados del análisis bibliográfico sobre uso del hábitat de la cabra montés y otras especies del género Capra se ha considerado que los principales factores que definen un hábitat adecuado para la cabra montés en la Sierra de Ancares son:

- Las zonas de refugio, definidas como los relieves abruptos con elevada pendiente y presencia de roca. A raíz de las experiencias recogidas sobre otras poblaciones de cabra montés, se ha determinado las áreas de refugio como una superficie circundante a las zonas de cortados rocosos 
(García-Serrano et al., 2010). Estos cortados se han estimado mediante una combinación de pendientes elevadas (superiores al 80\%) y elevada rugosidad del terreno (curvatura total superior a 0,05 radianes $\cdot \mathrm{m}^{-2}$ ). Estas zonas se reparten en la RNCA entre algunos afloramientos rocosos de las crestas y laderas de los principales valles.

- La orientación, a partir de la existencia de zonas de invernada con orientaciones sur y este que reciban una mayor insolación.

- Las interacciones con el hombre, considerando áreas molestas (carreteras y núcleos de población habitados), e indiferentes el resto.

- La conectividad entre distintas áreas de refugio del macizo. En este sentido se ha valorado positivamente la cercanía al eje central que comunica con la vertiente leonesa.

A partir de los mapas resultantes de representar las variables descritas se ha realizado una zonificación general, agrupando las zonas más adecuadas ajustando su superficie en función de la orografía con la ayuda de ortofotografías. Se han establecido tres categorías de calidad de hábitat (Figura 2):

- Zona A. Calidad media-alta. Pequeñas zonas de roquedos cercanas entre sí formando una unidad de cientos de hectáreas, con algunas zonas de refugio en las solanas, alejadas de núcleos de población y carreteras, y conectadas con la vertiente leonesa. Suman 1.250 ha.

- Zona B. Calidad media-baja. Terrenos con pequeñas zonas de refugio conectadas entre sí pero sin conexiones con otras zonas favorables más extensas, situados en zonas bajas, exclusivamente en las umbrías o cercanas a focos de molestias humanas como pueblos y carreteras. Suman 1.200 ha.

- Zona C. Calidad Baja. Zonas que no ofrecen áreas de refugio o estas son muy pequeñas y aisladas. Zonas con alta densidad de núcleos de población. Terrenos alomados en general con un paisaje poco quebrado y sin presencia de roquedos importantes. Ocupan la mayor parte de la RNCA, aproximadamente 5.500 ha.

Según las características descritas para caracterizar el hábitat de la cabra montés la vertiente leonesa presentan el hábitat de mayor calidad del macizo con grandes superficies de roquedos, pendientes pronunciadas con orientaciones de solana y alejadas de núcleos de población y carreteras. 


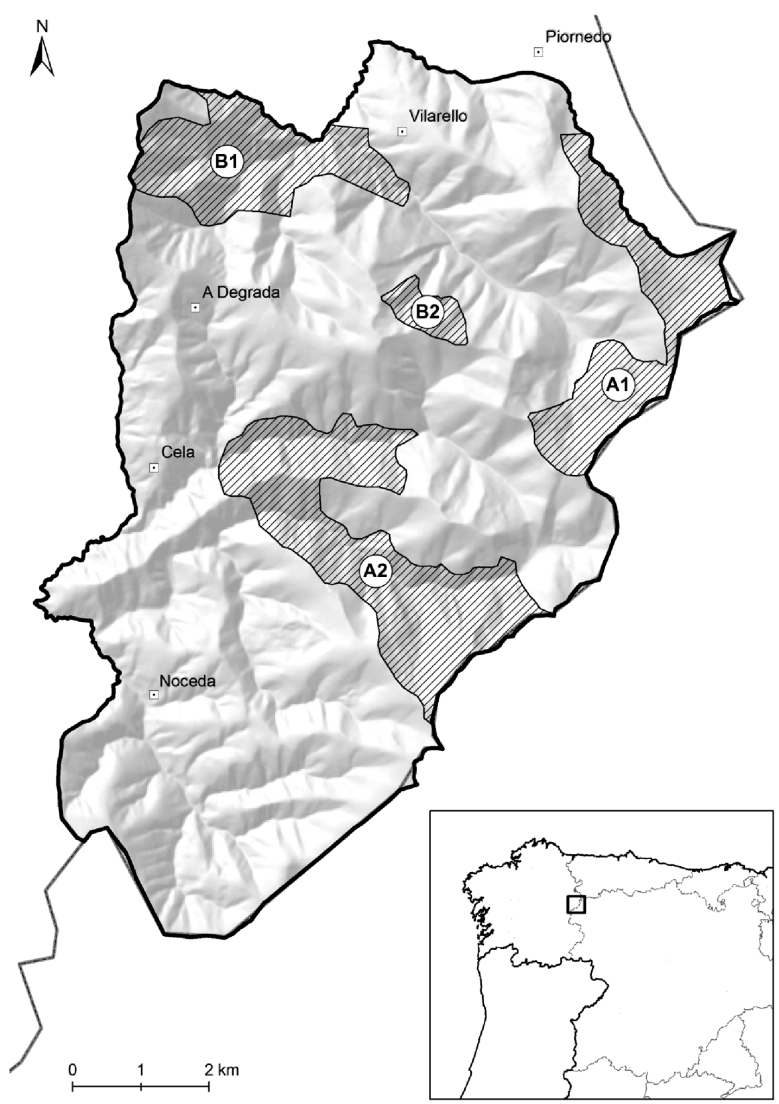

Figura 2. Calidad de hábitat de la RNCA para la cabra montés. Zonas de calidad media-alta: A1 (cuerda Pena Rubia-Pena Longa-Serra de Villous), A2 (Valles de Brego y Vara). Zonas de calidad media-baja: B1 (Chao do Teso-Valle de Ortigal) y B2 (Zona alta del Valle de Ortigal). Zonas de calidad baja: resto de la RNCA.

Figure 2. Habitat quality for Iberian wild goat in the National Game Reserve of Os Ancares. Areas of highmedium quality: A1 (Pena Rubia-Pena Longa-Serra de Villous ridge), A2 (Brego and Vara valleys). Areas of médium-low quality: B1 (Chao do Teso- Ortigal valley) y B2 (high part of the Ortigal valley). Areas of low quality: rest of the National Game Reserve of Os Ancares.

\section{Discusión}

Las poblaciones de ungulados silvestres han aumentado en toda Europa en las últimas décadas (Gortázar et al., 2000, Apollonio et al., 2010). Esto ha propiciado el desarrollo de métodos de estima poblacional y herramientas de 
análisis espacial, que han permitido que la gestión de sus poblaciones pueda llevarse a cabo con una base técnica y científica (Nores et al., 2007). En este sentido la mayor parte de los datos cartográficos utilizados en este trabajo han contado con una resolución adecuada, teniendo en cuenta la escala a la que se ha trabajado.

En los Ancares existe en la actualidad una población de cabra montés que se encuentra en expansión y que previsiblemente ampliará su área de distribución hasta ocupar todas las áreas con hábitat favorable de ambas vertientes del macizo, haciendo un uso estacional o temporal de muchas de ellas.

La variable limitante del hábitat la constituye la presencia de zonas de refugio, esto es roquedos con pendiente; valorando positivamente su localización en zonas de solana, la superficie de las zonas, su conexión y su distancia a carreteras y núcleos de población. El hábitat favorable es escaso, fragmentado y ocupa sobre todo las umbrías de los valles laterales. Alados y Escós (1996) apuntan que una densidad de 2 a 3 individuos $\mathrm{km}^{-2}$ es la más adecuada para que el índice de reproducción de las poblaciones y la tasa de supervivencia de las crías sean más elevadas. Con estos datos el número de individuos que podría albergar la RNCA sería de 40, aunque teniendo en cuenta la presencia de una población numerosa en vertiente leonesa y la movilidad de la especie, este número puede sufrir importantes variaciones estacionales. Sin embargo la densidad total de ungulados, domésticos y salvajes, ofrece una visión más realista de la situación. La principal especie que comparte el espacio con la cabra es el rebeco, que mantiene unas densidades elevadas en la zona (A. Martino com. pers.). La población oriental de rebeco cantábrico está severamente afectada por sarna Sarcoptes scaibiei, que afecta también a la cabra montés en Riaño (González-Quirós y Solano, 2009). El rebeco tiene una distribución continua por la Cordillera Cantábrica, así que existe riesgo de propagación a otros lugares. Esto, unido a la escasez de pastos en las zonas que comparten rebecos y cabras, debido al intenso proceso de matorralización (Rebollo y Gómez-Sal, 1996), incrementa la posibilidad de contacto entre individuos y de transmisibilidad de la enfermedad.

Es importante recalcar la importancia de las reservas de caza en la recuperación de especies cinegéticas en general, y de la cabra montés en la Cordillera Cantábrica en particular. Estos territorios cinegéticos de gestión pública, declarados en terrenos con excepcionales potencialidades cinegéticas, se crearon para fomentar la caza, controlar del furtivismo, fomentar el turismo cinegético y, sobre todo, recuperar las poblaciones de especies cinegéticas en un momento en el que muchas de ellas tenían diezmadas sus poblaciones (Pita et al., 2012). Actualmente han cumplido sobradamente los objetivos por los que fueron declaradas, la mayoría de las poblaciones animales que justificaban su interés se han recuperado y a partir de ellas han iniciado la colonización de 
otros territorios. Este podría ser el caso de la cabra montés en la provincia de Lugo, donde existen otras áreas cercanas a la RNCA aparentemente con buenas potencialidades de hábitat para albergar poblaciones de cabra montés como es el caso del Balouta o el Ser.

En definitiva, argumentos como la escasez de zonas de hábitat adecuado, los fracasos de sueltas previas y la existencia de una población en aumento en la vertiente leonesa de la sierra desaconsejan realizar un refuerzo poblacional. Sin embargo la voluntad de incrementar la variabilidad genética de la cabra montés en la Cordillera Cantábrica, que ha sufrido previamente varios cuellos de botella sucesivos, podría justificar esta actuación. En este sentido la zona más adecuada para la suelta sería la que mantiene un grupo de hembras estable dentro de la RNCA. En cualquier caso es importante aplicar una visión de macizo a la gestión de los ungulados de montaña de Ancares, con la consiguiente necesidad de coordinación territorial (Herrero et al., 2010), entre otras cosas porque la gestión de unidades exclusivamente administrativas, presenta serias dificultades a la hora de evaluar los resultados obtenidos (Pañella et al., 2010).

\section{Agradecimientos}

José Enrique Díaz Colmenero, Andrés Rodríguez López y José Amigo López, guardas de la RNCA, nos facilitaron abundante información sobre la situación actual de la reserva y su historia y nos acompañaron durante el trabajo de campo. Juan Carlos Peral, Director de las RRC de León, nos facilitó abundante documentación sobre la distribución de la cabra montés por la Cordillera Cantábrica; y Andrés Martino, guarda de la RRCA, nos aportó importantes datos sobre el origen y la distribución actual de las cabras monteses en Ancares. Jorge Fernández Layna, consultor especializado en biología de la conservación y buen conocedor de la RNCA, nos aportó su experiencia y visión sobre la comunidad de grandes mamíferos. Carlos Nores, profesor titular del Departamento de Zoología de la Universidad de Oviedo, nos aportó bibliografía histórica sobre la especie en Galicia y la Cordillera Cantábrica. A todos ellos nuestro más sincero agradecimiento. Este trabajo es fruto de una asistencia técnica financiada por la Xunta de Galicia y dirigida por Antonio Callejo, director de la Reserva de Caza de Os Ancares. 


\section{PRADA \& J. HERRERO}

\section{Referencias}

Alados, C.L. \& Escós J., 1996. Ecología y comportamiento de la cabra montés: consideraciones para su gestión. Museo Nacional de Ciencias Naturales. Monografía n $\mathrm{n}^{\mathrm{1}}$ 11, 329 pp., Madrid.

Almança, C., 1992. Notes on Capra pyrenaica lusitanica Schlegel, 1872. Mammalia, 56: 121-124.

Amills, M., Jiménez, N., Jordana, J., Riccardi, A., Fernández-Arias, A., Guiral, J., Bouzat, J.L., Folch J. \& Sánchez, A., 2004. Low diversity in the major histocompatibility complex class II DRB1 gene of the Spanish ibex, Capra pyrenaica. Heredity, 93: 266-272.

Apollonio, M. Andersen, R., \& Putman, R., 2010. Ungulate management in Europe in the XXI Century. Cambridge University Press. Cambridge.

Arribas, Ó., 2004. Fauna y paisaje de los Pirineos en la Era Glaciar. Lynx Ediciones, Bellaterra. 540 pp.

Byers, J. A., 1977. Terrain preferences in the play behavior of Siberian ibex kids (Capra ibex sibirica). Z. Tierpsychol., 45: 199-209.

Cabrera, A., 1911. The subespecies of the Spanish ibex. Proceed. Zool. Soc.London, 66: 963-977.

Cabrera, A,. 1914. Fauna Ibérica. Mamíferos. Museo Nacional de Ciencias Naturales, Madrid. 441 pp.

Cano-Manuel, J., Castillo, A., Granados, J. E., Fandos, P., Pérez, J. M., Alasaad, S., Sarasa, E., Serrano, E., Moço, G., \& Soriguer, R. C., 2008. Nuevas tecnologías aplicadas al seguimiento de cabra montés en el Parque Nacional de Sierra Nevada: collares GPS-GSM. En: Tendencias actuales en el estudio y conservación de los Caprinos Europeos. Ed: Junta de Andalucía. Consejería de Medio Ambiente. Granada. España.

Choisy, J.P., 1994. Reintroduction de bouquetins Capra sp.: conditions de réussite, choix des massifs, enseigments. L'example du Vercors. Ibex, 1: 1533.

Clouet, M., 1979. Note sur la systématique du bouquetin d'Espagne. Bulletin de Society Historie Naturelle de Toulouse, 115: 269-277.

Conde de Yebes, 1943. Veinte años de caza mayor. Espasa-Calpe, Madrid, 319 pp.

Couturier, M., 1962. Le bouquetin des Alpes (Capra aegagrus ibex ibex L.). Ed. Par l'auteur, Grenoble, 1.564 pp.

Crampe, J.P., 1991. Le bouquetin iberique. Elements pour une réintroduction au versant Nord des Pyrénées Occidentales. Documents Scientifiques $d u$ Parc National des Pyrénées, 26: 1-187.

Escós, J. \& Alados. C. L., 1992. Habitat preference of Spanish ibex and other ungulates in Sierras de Cazorla y Segura (Spain). Mammalia, 56(3): 393-406. 
Fandos, P., 1991. La cabra montés (Capra pyrenaica) en el Parque Natural de Cazorla, Segura y Las Villas. Colección Técnica, Icona, Madrid, 176 pp.

Fandos, P., Buron D., \& Granados, J. E., 2007. La cabra montes en España. Revista Trofeo. Diciembre 2007.

Fox, J.L., Sinha, S. P., \& Chundawat, R. S., 1992. Activity patterns and habitat use of ibex in the Himalaya Mountains of India. Journal Mammalia, 73(3): 527-534.

Francisci, F., Focardi, S., \& Boitani, L., 1985. Male and female Alpine ibex: phenology of space use and herd size. En: S. Lovari (Ed): The biology and management of mountain ungulates. Croom Helm, Londres: 124-133 pp.

García-González, R. \& Cuartas, P., 1989. A comparison of the diets of the wild goat (Capra pyrenaica), domestic goat (Capra hircus), mouflon (Ovis musimon) and domestic sheep (Ovis aries) in the Cazorla mountain range. Acta Biologica Montana, 9: 123-132.

García-González, R. \& Cuartas, P., 1992. Feeding strategies of Spanish wild goat in the Cazorla Sierra (Spain). Ongules/Ungulates, 91: 167-170.

García-González, R. \& Herrero, J., 1999. El bucardo de los Pirineos: historia de una extinción. Galemys, 11 (1): 17-26.

García-González, R., 2011. Elementos para una filogeografía de la cabra montés ibérica (Capra pirenaica Schinz, 1838). Pirineos, 166: 87-122.

García-Serrano, A., Prada, C., Fernández-Arberas, O., \& Herrero, J., 2010. Seguimiento de la cabra montés Capra pyrenaica en Zaragoza durante 2010. Informe interno del Gobierno de Aragón.

Gauthier, D., Chatain, G., Choisy, J. P. Crampe, J. P. Martinot, J. P., Michallet, J., Terrier, G., Tron, L. \& Villaret, J. C. 1994. L'organisation des réintroductions de bouquetin en France. La charte du bouquetin. Ibex, 1: 114.

Gonzalez, G., 1982. Eco-ethologie du bouquetin en Sierra de Gredos. Acta Biologica Montana, 1: 177-215.

González-Quirós, P. \& Solano, S., 2009. Monitorización del brote de sarna sarcóptica en las reservas de caza regionales de Asturias. En: F.J. Barbería y B. Palacios, editores: El Rebeco Cantábrico Rupicapra pyrenaica parva. Conservación y Gestión de sus poblaciones: 292-319 pp.

Gortázar C., Herrero J., Villafuerte R. \& Marco J., 2000. Historical examination of the status of large mammals in Aragon, Spain. Mammalia, 64: 411-422.

Granados, J.E., Serrano, E., Pérez, M. C., Fandos, P., Weykam, S. \& Soriguer R. C. 2003. Caracterización del hábitat ocupado por la cabra montés (Capra pyrenaica, Schinz 1838) en Andalucía. En: J.M. Pérez (Ed.): “In memoriam al Prof. Dr. Isidoro Ruiz Martínez". Universidad de Jaén. Jaén: 391-404 pp.

Hernández Pacheco, E. 1919. La caverna de la Peña de Candamo (Asturias). Museo de Ciencias Naturales de Madrid. 
Herrero, J. \& Pérez, J. M., 2008. Capra pyrenaica. In: IUCN 2010. IUCN Red List of Threatened Species. Version 2010.4. www.iucnredlist.org.

Herrero J., Garin, I., Prada, C. \& García-Serrano, A. 2010. Inter-agency coordination fosters the recovery of the Pyrenean chamois Rupicapra pyrenaica pyrenaica at its western limit. Oryx, 44(4): 529-532.

IGE 2010. Instituto Galego de Estatística. Padrón municipal. Xunta de Galicia http:/ / www.ige.eu

Llaneza L., García, E. J. \& Palacios, V., 2011. Seguimiento del lobo en los Ancares Lucenses. Informe interno de la Xunta de Galicia.

Manceau, V., Despres, L., Bouvet, J. \& Taberlet, P., 1999. Systematics of the Genus Capra Inferred from Mitochondrial DNA Sequence Data. Molecular Phylogenetics and Evolution, 13: 504-510.

Marqués de Valdueza, 1988. Tras las huellas del recuerdo. Memorias de un cazador veterano. Ed. El Viso. Madrid. 280 pp.

Martínez, T. \& Martínez, E. 1987. Diet of Spanish wild goat, Capra pyrenaica in spring and summer at the Sierras de Gredos, Spain. Mammalia, 35: 547-558.

Martínez, T., 1988a. Utilisation de l'analyse micrographique de fèces pour l'étude du régime alimentarie du boutequin de la Sierra Nevada (Espagne). Mammalia, 52: 46.

Martínez, T., 1988b. Données sur l'alimentation du bouquetin d'Espagne (Capra pyrenaica) dans la Sierra de Tejeda (Granada). Mammalia, 52, 2.

Martínez, T., 1989. Recursos tróficos de la cabra montés (Capra pyrenaica, Schinz, 1938) en la Sierra de Gredos, durante otoño e invierno. Ecología, 3: 179-186.

Martínez, T., 1992. Estrategia alimentaria de la cabra montés (Capra pyrenaica) y sus relaciones tróficas con los ungulados silvestres y domésticos en Sierra Nevada, Sierra de Gredos y Sierra de Cazorla. Universidad Complutense de Madrid. Tesis Doctoral.

Martínez, T., 1994. Hábitos alimentarios de la cabra montés (Capra pyrenaica) en zonas de distinta altitud en los puertos de Tortosa y Beceite. Referencia a la dieta de machos y hembras. Doñana Acta Vertebrata, 21: 25-37.

Moço, G., Guerreio, M., Ferreira, A. F., Rebelo, A., Loureiro, A., PetrucciFonseca, F. \& Pérez, J. M., 2006. The ibex Capra pyrenaica returns to its former Portuguese range. Oryx, 40: 351-354.

Nievergelt, B., 1966. DerAlpensteinbock (Capra ibex L.) in Seinem Lebenstraum. Mammalia Depicta. Verlag Paul Parey. Hamburg and Berlin, 85 pp.

Nores Quesada C. \& García-Rovés González, P., 2007. Libro Rojo de la Fauna del Principado de Asturias. Consejería de Medio Ambiente, Ordenación del Territorio e Infraestructuras del Principado de Asturias y Obra Social La Caixa, Oviedo. 
Ortuño, F. \& De la Peña, J., 1977. Reservas y Cotos Nacionales. 2-Región Cantábrica. Ed. Incafo, Madrid, 253 pp.

Palacios, F., Ibañez, Z., \& Escudero, J., 1978. Algunos datos sobre la alimentación de la cabra montés ibérica (Capra pyrenaica) y notas sobre la fauna de Montenegreto (Tarragona). Boletín de la Estación Central de Ecología, 7: 56-66.

Palomares, F. \& Ruiz-Martínez I., 1993. Status and conservation perspectives for the Spanish ibex population (Capra pyrenaica Schinz 1838) of Sierra Mágina Natural Park, Spain. Zeitschrift für Jagdwissenschaft, 39: 87-94.

Pañella, P, Herrero, J., Canut J., \& García-Serrano A., 2010. Long-term monitoring of Pyrenean chamois in a protected area reveals a fluctuating population. Hystrix It. J. Mamm. (n.s.) 21(2) 2010: 183-188.

Peracino, V. \& Bassano, B., 1994. Valutazione di idoneitá dei territori del Parco Naturale dell'Orsiera-Rocciavré al fini della reintroduzione dello stambecco (Capra ibex ibex, L.). Ibex, 1: 49-60.

Peral, J.C., 1993. La cabra montés vuelve a Riaño. Trofeo, 272: 70-76.

Pérez, J.M., Granados, J.E., Soriguer, R. C., Fandos, P., Márquez, F.J. \& Crampe J. P., 2002. Distribution, status and conservation problems of the Spanish ibex, Capra pyrenaica (Mammalia: Artiodactyla). Mammalia Revue, 32: 26-39.

Pérez, J.M., Granados J. E., \& Soriguer R. C., 1994. Population dynamic of the Spanish ibex Capra pyrenaica in Sierra Nevada Natural Park (Southern Spain). Acta Theriologica, 39 (3): 289-294.

Pfeffer, P., \& Settimo, R., 1973. Deplacements saisonners et competition vitale entre mouflons, chamois et bouquetins dans la Reserve du Mercantour. Mammalia, 37 (2): 201-219.

Pita M., Casas, S., Herrero, J., Prada C. \& García-Post, R, 2012. Game reserves in Spain: the public management of hunt. Forest Systems, 21 (3): 398-404.

Prada, C, García-Serrano, A., Fernández, O., Herrero J., 2010. Estima poblacional de cabra montés y muflón en la Reserva Valenciana de Caza Muela de Cortes. Informe interno de la Generalitat Valenciana.

Prada, C., Fernández, O., García-Serrano, A., \& Herrero, J., 2011. Seguimiento de la cabra montés en Castellón. Informe interno de la Generalitat Valenciana.

Rebollo S., \& Gómez-Sal, A., 1996. Relación entre la densidad de ungulados silvestres en pastizales de montaña de León y la evolución de la ganadería trashumante. En: Sierra Nevada. Conservación y Desarrollo Sostenible, V: 8991 pp. Actas de la Primera Conferencia Internacional Sierra Nevada Desarrollo Sostenible. Universidad de Granada.

San Segundo, M., \& Díez, L.F., 1997. Metodología para la selección de zonas de suelta de ejemplares de cabra montés (Capra pyrenaica victoriae) en la Reserva 
Natural del Valle de Iruelas (Ávila). Departamento de Ciencias Agroforestales. Escuela Técnica Superior de Ingenierías Agrarias. Palencia. 10 pp.

Seoane, V. L., 1866. Reseña de la Historia Natural de Galicia. Soto Freire, Lugo Uría, J., 1976. Los vaqueiros de Alzada y otros estudios de caza y etnografía. Biblioteca Popular Asturiana. Oviedo, 345 pp.

Wiersma, G., 1983. L'habitat saisonnier do bouquetin dans le Parc National de la Vanoise. Trav. Scient.Parc National Vanoise, XII: 211-222.

Wiersma, G., 1984. Seasonal use and quality assessment of ibex habitat. Acta Zoologica Fennica, 172: 89-90. 